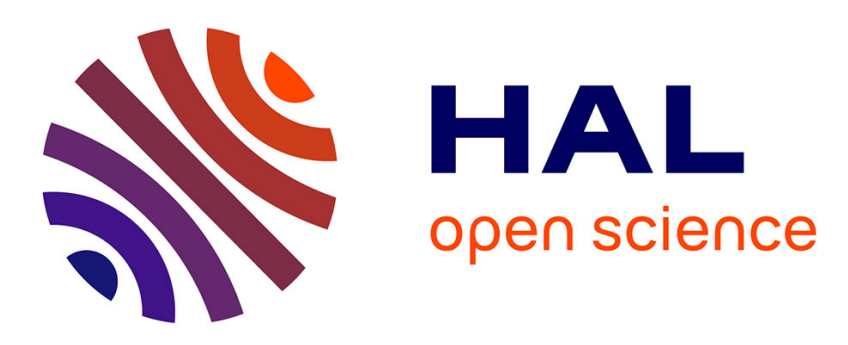

\title{
Buoyancy-aided convection flow in a heated straight pipe: comparing different asymptotic models
}

\author{
Walid Arfaoui, Mohamed Jomaa Safi, Pierre-Yves Lagrée
}

\section{To cite this version:}

Walid Arfaoui, Mohamed Jomaa Safi, Pierre-Yves Lagrée. Buoyancy-aided convection flow in a heated straight pipe: comparing different asymptotic models. Heat and Mass Transfer, 2015, pp.1. 10.1007/s00231-015-1677-1 . hal-01200663

\section{HAL Id: hal-01200663 \\ https://hal.sorbonne-universite.fr/hal-01200663}

Submitted on 16 Sep 2015

HAL is a multi-disciplinary open access archive for the deposit and dissemination of scientific research documents, whether they are published or not. The documents may come from teaching and research institutions in France or abroad, or from public or private research centers.
L'archive ouverte pluridisciplinaire HAL, est destinée au dépôt et à la diffusion de documents scientifiques de niveau recherche, publiés ou non, émanant des établissements d'enseignement et de recherche français ou étrangers, des laboratoires publics ou privés. 


\title{
Buoyancy-aided convection flow in a heated straight pipe: comparing different asymptotic models.
}

\author{
Walid Arfaoui ${ }^{\dagger o}$, Mohamed Jomaa Safi ${ }^{\dagger}$, Pierre-Yves Lagrée*o \\ $(\dagger)$ Ecole Nationale d'Ingénieurs de Tunis (ENIT) \\ BP 37, Le Belvédère 1002 Tunis, Tunisia \\ (*) CNRS, UMR 7190, Institut Jean Le Rond $\partial$ 'Alembert, \\ Boîte 162, F-75005 Paris, France \\ (o) Sorbonne Universités, UPMC Univ Paris 06, \\ Institut Jean Le Rond $\partial$ 'Alembert, \\ Boîte 162, F-75005 Paris, France \\ corresp author: Pierre-Yves Lagrée pyl@ccr.jussieu.fr
}

September 8, 2015

\begin{abstract}
A vertical straight circular adiabatic vertical long tube, open at its lower and upper ends, is heated at its base on a short portion. The flow is studied with the hypothesis of no pressure drop between the entrance and the exit. Direct resolution of Navier Stokes equations is done by finite volumes. The numerical solutions are then compared to a one dimensional model and to two asymptotic models. The first asymptotic model is inspired from boundary layer approximations whereas the second one is more a linear perturbation of the Navier Stokes Boussinesq equations. For moderate values of the Grashof number, pressure, starting from zero decreases over the heated part to a minimum and increases on the adiabatic tube to zero. For larger values of Grashof, a local maximum in pressure appears, this pressure hump may even be positive. The four model agree, for moderate Grashof. When increasing the Grashof, only the two asymptotic models recover the behavior obtained from the numerical simulations.
\end{abstract}

\section{Keywords}

Aiding mixed convection - Vertical open channel flows - Boundary conditions Boundary layer - Asymptotic models

\section{Nomenclature}

$G r$ Grashof (equivalent to $R e$ due to the choice of scales)

$g$ gravitational acceleration

$h$ heat transfer coefficient

$k$ thermal conductivity

$\ell$ length of the heated part 
$L_{c}$ length of the adiabatic channel

$\bar{p}$ deviation of pressure from hydrostatic pressure without dimension

$P e$ Péclet number

$r$ radial variable

$R$ radius of the channel

Re Reynolds number

$S$ section of the channel

$\bar{T}_{m}$ mean value of the temperature across a section ponderated by the Poiseuille solution

$T_{0}$ reference temperature

$T_{w}$ temperature of the heated part of the channel

$u$ velocity along the tube

$\bar{U}_{\text {max }}$ maximum of the velocity without dimension

$\bar{U}$ mean value of the velocity across a section

$U_{0}=\left(R^{2} \rho g \alpha\left(T_{w}-T_{0}\right)\right) / \mu$ velocity scale

$v$ radial velocity across the tube

$x$ axial direction along the tube

\section{Greek symbols}

$\alpha$ coefficient of thermal expansion

$\Delta T=\left(T_{x}-T_{0}\right)$ driving difference of temperature

$\lambda$ a thermal length for the $1 \mathrm{D}$ model

$\mu$ dynamic viscosity

$\nu$ kinematic viscosity

$\rho_{0}$ reference density of the fluid

\section{Superscripts}

$-{ }^{\wedge} \sim$ quantity without dimension

\section{Introduction}

The starting point of this study is to simulate the flow in the solar towers. Those devices are huge towers $(100 \mathrm{~m}$ to $1 \mathrm{~km})$, at the base of the tower the air is heated by the walls themselves heated by the sun (see figure 1 for a rough sketch). This results in a free convection flow, which aspires fresh air at the base of the tower so that a mixed convection settles. As a turbine is disposed in the tower, electricity is generated. Those devices are believed to be a response to fossil energy crisis, and produce no carbon. Erected in a desert, they may produce sustainable energy.

Most of the papers (devoted to solar towers, for instance [32, 31, among others) deal with turbulent k-epsilon with Boussinesq buoyancy in a simple geometry (radiation results only in a heated wall, called "collector"). Then, often in the literature, a simple one dimensional (1D) approach is proposed [17, 16, 4, 33, always with compressible equations (even if Navier Stokes are mainly solved in incompressible framework). Our approach mimics this flow by a buoyant laminar flow. Effectives towers have a rather complicated shape, and shape effects are believed to play a role (local acceleration of the flow...). Here, we will simplify the geometry and only look at a simple tube of circular 
section. Anyway, the aim of this paper is absolutely not to design and optimize a power plant (furthermore, the pressure drop due to the electrical generator is not modeled). In fact, this kind of problem may have application in buildings cooling as well. Our scope is to over simplify the set up and model it just as a straight tube with no pressure at the entrance and at the exit and to propose some models to analyze the laminar flow obtained. The equations are the steady laminar Navier Stokes with Boussinesq approximation with no radiation and no conduction in the walls.

We want to propose here, first a $1 \mathrm{D}$ model really consistent with the $2 \mathrm{D}$ axi Navier Stokes Boussinesq incompressible proposed resolution, and second, we want to improve the description of the flow in the tube by other more sophisticated asymptotic models. As the longitudinal scale is larger than the transverse scale, it is natural to think that the Graetz problem [29, 25] and Lévêque problems 26] are central to this analysis (see [20] for perturbations of Lévêque problem). They correspond to asymptotically large Reynolds and Péclet numbers. Hence, we will present boundary layer (or Prandtl) equations to analyze the flow which is a free convection flow (see 22 for mixed convection equations and problems of parabolicity). Another asymptotic description that we will propose, consists to consider the flow as a perturbation of a Poiseuille flow, so a linearized Navier Stokes system will be presented (corresponding to asymptotically small heating).

One of the focus of this study will be on the pressure field plotted along the centerline of the pipe, it is seldom displayed (Le Quéré [18] and Desrayaud et al. [6]). The reason for our interest is that the pressure deviation from the hydrostatic pressure is zero (at least in solar chimneys with a large round collector). It is then paradoxal that a flow exists with no pressure gradient. All aver the paper, the term "pressure" refers to the pressure deviation from the hydrostatic pressure as it is classical in Boussinesq approximation. A first response to this paradox is that the buoyancy drives the flow, nevertheless, most of the pipe is submitted to an adverse pressure gradient. Furthermore in plotting the pressure curves, for moderate Reynolds, the pressure decreases linearly smoothly from zero at the entrance (favorable pressure gradient), and after a minimum, increases linearly smoothly to zero again at the output (adverse pressure gradient). But, we will see that the variations are not so monotonic for large enough Reynolds. The pressure curve presents a hump, or even a positive maximum. We aim to explain this with asymptotics. The reason is that we will see it is not possible to explain this with the 1D theory, and that, between full Navier Stokes and 1D theory there exists place for some asymptotic models which will be presented. The main contribution of this paper is this comparison between full NS and different simplified models, each one simplifies some aspects of the flow. Furthermore, in the discussion, we will mention that any other boundary condition in pressure like a Bernoulli one (Garnier et al. [12]) can be applied in the asymptotic model.

The paper is organized as follows, first the model equations with and without dimension (section 2). Then the numerical resolution with a focus on the pressure. In section 3, we present the most classical models, Poiseuille, Graetz and Lévêque, and the $1 \mathrm{D}$ approach (section 3.2). Then, to circumvent the drawbacks of the 1D approach two asymptotic models are presented (with dif- 
ferent asymptotic approximations in 3.3 and 3.4). Finally (section 4 ) we discuss the pertinence of all those models compared to the numerical solution of Navier Stokes and how those model can help the analysis of the flow.

\section{Direct numerical simulation of the problem}

\subsection{Formulation of the problem in the Boussinesq approx- imation framework}

The geometry (see figure 1 right) is a straight tube of circular cross section. The heated part is called collector, the adiabatic part, chimney (this terminology is reminiscent of the solar tower just for convenience). The flow is assumed to be axi-symmetric, laminar, steady, incompressible, and with no dissipation in the heat equation. There is no radiation in the flow and in the wall and no conduction in the wall in this model. Thermo-physical properties of the fluid are assumed to be constants ( $\rho_{0}$ density, $\nu$ kinematic viscosity, $k /\left(\rho_{0} c_{p}\right)$ thermal diffusivity). The buoyancy force is modeled by the Boussinesq approximation so that the flow is incompressible with a body force proportional the difference between the local temperature $T$ and the reference temperature $T_{0}$ ( $\rho$, the density, is $\left.\rho_{0}\left(1-\alpha\left(T-T_{0}\right)\right)\right)$. The reference temperature is the external constant temperature, the exterior is supposed infinite, steady, at hydrostatic pressure and is supposed to not perturb the tube. The Boussinesq approximation allows to define a pressure $p$ which is the effective pressure reduced by the hydrostatic pressure $\rho_{0} g x$. Hence, the flow is described by the Navier Stokes equations with heat equation and Boussinesq approximation. Boundary conditions are no slip at the wall $\vec{u}(x, r=R)=0$, prescribed temperature at the collector wall $T(-\ell<x<0, r=R)=T_{w}$ and no flux in the adiabatic chimney $\partial T / \partial r=0$ for $L_{c} \geq x>0$ and $r=R$. Entrance and output condition are not so simple for a real setup. Near the entrance the fluid is aspirated, and at the output of the chimney, a buoyant jet develops. In the chosen configuration (see figure 1), we look at the straight pipe alone, as the external air is at hydrostatic pressure, the deviation of pressure is zero at the input and at the output. So we impose $p=0$ at the entrance $x=-\ell$ and at the exit $x=L_{c}$ the pressure is zero again: $p=0$ (the velocity is such that $\partial_{x} u=0$ at the input and the output). Taking into account the region surrounding the entrance and the effect of the buoyant jet formation is assumed to be negligible. The final boundary conditions are on figure 1 right. Those boundary conditions define a model problem that we want to solve by different methods, first with direct simulation and then with some assumptions we will define some asymptotic problems.

\subsection{Problem without dimension}

To solve the problem, we have to write it without dimensions. Let us then consider the radius $R$ as a characteristic length for the flow, $\Delta T=T_{w}-T_{0}$ a reference temperature difference. Since the present work deals with free convection, there is no prescribed characteristic velocity scale in the problem. However, from balancing diffusion and buoyancy in the momentum equation a velocity scale (say $U_{0}$ ) can be derived to be $U_{0}=\frac{g \alpha \Delta T R^{2}}{\nu}$. This balance is pertinent in the chimney (far from the collector) where the buoyancy drives the flow which 
is damped by viscosity. This makes the Reynolds number $\left(R e=U_{0} R / \nu\right)$ to be identical to the Grashof number $\left(G r=\frac{g \alpha \Delta T R^{3}}{\nu^{2}}\right)$, so $R e=G r$. The dimensionless variables become then:

$$
\bar{x}=\frac{x}{R}, \bar{r}=\frac{r}{R} \bar{u}=\frac{u}{U_{0}}, \bar{v}=\frac{v}{U_{0}}, T=T_{0}+\Delta T \bar{T}, \bar{p}=\frac{p-p_{0}}{\rho_{0} U_{0}^{2}} .
$$

The non-dimensional general form of the differential equations describing the steady flow are given by:

$$
\begin{gathered}
\frac{1}{\bar{r}} \frac{\partial \bar{r} \bar{v}}{\partial \bar{r}}+\frac{\partial \bar{u}}{\partial \bar{x}}=0 \\
\bar{v} \frac{\partial \bar{v}}{\partial \bar{r}}+\bar{u} \frac{\partial \bar{v}}{\partial \bar{x}}=-\frac{\partial \bar{p}}{\partial \bar{r}}+\frac{1}{R e}\left(\frac{1}{\bar{r}} \frac{\partial}{\partial \bar{r}}\left(\bar{r} \frac{\partial \bar{v}}{\partial \bar{r}}\right)+\frac{\partial^{2} \bar{v}}{\partial \bar{x}^{2}}\right) \\
\bar{v} \frac{\partial \bar{u}}{\partial \bar{r}}+\bar{u} \frac{\partial \bar{u}}{\partial \bar{x}}=-\frac{\partial \bar{p}}{\partial \bar{x}}+\frac{1}{R e}\left(\frac{1}{\bar{r}} \frac{\partial}{\partial \bar{r}}\left(\bar{r} \frac{\partial \bar{u}}{\partial \bar{r}}\right)+\frac{\partial^{2} \bar{u}}{\partial \bar{x}^{2}}\right)+\frac{1}{R e} \bar{T} \\
\bar{v} \frac{\partial \bar{T}}{\partial \bar{r}}+\bar{u} \frac{\partial \bar{T}}{\partial \bar{x}}=\frac{1}{R e P r}\left(\frac{1}{\bar{r}} \frac{\partial}{\partial \bar{r}}\left(\bar{r} \frac{\partial \bar{T}}{\partial \bar{r}}\right)+\frac{\partial^{2} \bar{T}}{\partial \bar{x}^{2}}\right) .
\end{gathered}
$$

Boundary conditions are no slip at the wall $\bar{u}(\bar{x}, \bar{r}=1)=0, \bar{v}(\bar{x}, \bar{r}=1)=0$, prescribed temperature in the collector $\bar{T}(-\bar{\ell}<\bar{x}<0, \bar{r}=1)=1$ at the entrance $\bar{T}(\bar{x}=-\bar{\ell}, \bar{r})=0$ (with $\bar{\ell}=\ell / R$ ). There is no flux in the adiabatic chimney $\partial \bar{T} / \partial \bar{r}=0$ in $0<\bar{x}<\bar{L}_{c}$ and $\bar{r}=1$ (with $\bar{L}_{c}=L_{c} / R$ ).

The chosen configuration is with $\bar{p}=0$ at the entrance $\bar{x}=-\bar{\ell}$ for any $\bar{r}$, and at the output $\bar{x}=\bar{L}_{c}$ the pressure is zero again: $\bar{p}=0$ (for any $\bar{r}$ ). Note that we impose $\partial \bar{T} / \partial \bar{x}=0, \partial \bar{u} / \partial \bar{x}=0$ and $\bar{v}=0$ at the entrance and the exit. We have to note that the velocity at the entrance (hence the velocity flux) is a result of the computation as the pressure drop is given.

In other words, one of the questions of the problem is: what is the relation between the computed velocity flux (without dimension) in the tube and the non dimensional parameters of the problem $\bar{\ell}, \bar{L}_{c}, \operatorname{Pr}$, and $\operatorname{Re}$ ?

\subsection{Numerical resolution}

To asses this question, the numerical resolution is performed using a Navier Stokes solver (the free solver Gerris [28, 14]). Finite volumes are used for discretization. A projection method is used for incompressibility leading to a Poisson equation for pressure. A multigrid solver is used to solve this Poisson equation, (some details may be found in [24]). The boundary conditions are those of the previous section (zero pressure at the input/ output, Neumann boundary condition equal to zero on the wall and axis of symmetry). The viscous step for velocity is implicit (Neumann at the input/ output, Dirichlet zero on the wall). The unsteady equations are solved up to the steady flow, the criteria of convergence is $5 \mathrm{e}-7$ on velocity. The mesh has been changed by powers of 2 , $\Delta \bar{x}=\Delta \bar{r}=2^{-N}$ where $\mathrm{N}$ ranges from 2 to 8 (see figure 4 ). The pertinence of discretization, is that details in Lévêque solution and Graetz solution are well reproduced on figure 7 and 8 . As a rule of thumbs, the Lévêque thermal boundary layer at $R e=5000$ is $R e^{-1 / 3} \simeq 0.058$, this is nearly equal to $2^{-4}=0.0625$, so that we have enough points in our simulations.

The problem is characterized by the geometry $\bar{\ell}, \bar{L}_{c}$ and by the physical characteristics of the fluid $R e=G r$ and $P r$. Any of those quantities may be 
changed, the result of the computation are the temperature, velocities and pressure fields (with no dimension). We will focus on configurations where $\bar{\ell}=O(1)$ (the heated part of the pipe is from one to five radius length) and where $\bar{L}_{c} \gg 1$ (the chimney is longer than the radius, in practice from 10 to 100). We will explore values of $R e$ from small $(10)$ to moderate $\left(10^{4}\right)$. For larger values the flow may become unsteady and transitional. Note that the computations are done for $\operatorname{Pr}=1$ (the "model fluid"), Rayleigh, Grashof and Reynolds numbers are then equal.

The flow is solved unsteady in time up to a steady configuration (this steady configuration no more exists for $R e \gtrsim 5000$, we will not study this unsteady régime). At small time, in the collector the flow is heated at the wall, this promotes free convection. In the chimney, the wall thermal free convection boundary layer merges and the temperature is finally uniform across the pipe. This promotes by mass conservation an aspiration from the entrance and the result is to a first approximation a Poiseuille flow. The effect of the driving temperature is so large that the flows climbs up. The flow is then finally steady and the final problem the flow is generated by the heated part of the tube.

The mean temperature (across $\bar{r}$ ) increases in the collector and is then constant in the adiabatic part. If the tube is large enough, the temperature itself is constant (the temperature does not change in $\bar{r}$ nor in $\bar{x}$ ) as well in the chimney.

The velocity remains more or less parabolic in shape: $\bar{u}$ is proportional to $\left(1-\bar{r}^{2}\right)$ and $\bar{v} \simeq 0$ except near the $\bar{x}=0$ as the temperature change promotes changes in velocity. This can be seen on figure 4 where the center velocity is displayed, it remains at first approximation nearly constant.

The more interesting thing is the behavior of the pressure in the tube. The pressure decreases from zero in the collector due to the flow in it (which is again close to Poiseuille). Then the pressure increases in the chimney (with the same Poiseuille flow, the buoyancy acting as a constant pressure gradient). The pressure is zero at the exit. All the tube is in depression $\bar{p}<0$ everywhere for moderate values of $R e$.

The change of pressure is smooth for $R e$ smaller than say 500: the pressure is linear with a negative slope for $\bar{x} \lesssim 0$ and positive slope for $\bar{x} \gtrsim 0$. This gentle behavior is observed on figure 4 where three different collector lengths are displayed and four values of $R e$ are displayed. The same is plotted on figure 5 but for a fixed collector length and three different chimney lengths. We plot $\operatorname{Re} \bar{p}(\bar{x}, \bar{r}=0)$ as it is clearly the good scale for the pressure in the chimney. This comes from the balance of pressure gradient, viscous dissipation and thermal buoyancy in equation 4 .

But for larger values of $R e$, a small hump appears in the distribution of pressure along the axis at the junction collector/ chimney $(\bar{x}=0)$. This may be observed on figure 5 where the last $R e=1000$ presents this change in the slope of pressure. Increasing the collector length increases this hump, as well as increasing $R e$. This may even lead to strong adverse pressure gradient around $\bar{x} \simeq 0$, with a positive maximum for pressure. After this maximum, the pressure decreases in the chimney and reincreases to the exit. This is displayed on figure 6 where we clearly see negative, slightly positive an again negative pressure along the axis.

In the following, we will present simplified models which try to reproduce and explain those behaviors. 


\section{Analysis of the solution, simplified models}

\subsection{Simplified analysis with Lévêque and Graetz}

\subsubsection{Basic flow}

To analyse the flow, one has to be aware of two simple asymptotic solutions for thermal flows in pipes (here $\left(\ell+L_{c}\right) \gg R$ ). Obviously, from the numerical laminar simulation at moderately high Reynolds numbers, presented in the previous section, one sees clearly that the velocity profile is close to a Poiseuille profile (the maximum velocity is related to the pressure gradient):

$$
u=U_{\max }\left[1-(r / R)^{2}\right], \quad U_{\max }=\left[\frac{1}{4 \mu}\left(-\frac{d P}{d x}\right)\right] R^{2}
$$

So that, at first glance, if one neglects the buoyancy, the problem is a thermal response to a discontinuity in the conditions of temperature in a Poiseuille flow. When the Péclet number is large, and the tube long, this problem is well known, the model problem is the Graetz problem ([29]). Not so far from the discontinuity of temperature, the problem is known as Lévêque problem ([26]), see [27] for a discussion.

One of the questions is then: for $\bar{\ell}=O(1)$ and $\bar{L}_{c} \gg 1$ and $R e$ from small to enough large, how the maximal velocity $\bar{U}_{m}$ of the entrance Poiseuille profile $\bar{U}_{m}\left(1-\bar{r}^{2}\right)$ changes? In other words: what is the relation between $\bar{U}_{m}$ and the parameters of the problem $\bar{\ell}, \bar{L}_{c}$, and $R e$ ? We will try to respond to this using asymptotic models.

\subsubsection{The Lévêque problem}

In the collector part, for $-\bar{\ell}<\bar{x}<0$, the cold temperature $\bar{T}$ from the outside fluid experiences a unit heating. The flow has a Poiseuille profile, without dimension: $\bar{U}_{p}=\bar{U}_{\max }\left(1-\bar{r}^{2}\right)$. The $\bar{U}_{\max }$ is introduced for convenience and will be clarified thereafter. The Reynolds number is large (Péclet number defined as $\left.P e=R e_{\max } P r\right)$, so that a Matched Asymptotic Expansion with $\varepsilon \ll 1$ is introduced near the wall where the flow is heated. In the vicinity of the wall $(\bar{r}=1-\varepsilon \hat{r}$.$) , an asymptotically thin thermal boundary layer develops. Near the$ wall, the Poiseuille profile reduces to a shear flow $\left(u=2 \varepsilon \hat{r}+O\left(\varepsilon^{2}\right)\right)$. So that by dominant balance $\varepsilon^{3}=1 /\left(2 \bar{U}_{\max } P e\right)$ the heat equation reduces to its two dominant terms, the convection in a shear flow and the transverse diffusion:

$$
\hat{r} \frac{\partial \hat{T}}{\partial \bar{x}}=\frac{\partial^{2} \hat{T}}{\partial \hat{r}^{2}}
$$

The boundary condition for the wall of the tube are given by $\hat{T}(\bar{\ell}<\bar{x}<0,0)=1$ $\hat{T}(\bar{x}=\bar{\ell}, \hat{r})=0$, the matching boundary condition gives

$$
\hat{T}(x, \hat{r} \rightarrow \infty)=\bar{T}(x, \bar{r} \rightarrow 0)
$$

so that $\hat{T}(x, \infty)=0$. This problem has the well known self similar solution $\hat{T}=\theta\left(\hat{r} / \bar{x}^{1 / 3}\right)$, with $\theta(\eta)=\Gamma\left(1 / 3, \eta^{3} / 9\right) / \Gamma(1 / 3)$, were $\Gamma(s, x)=\int_{x}^{\infty} t^{s-1} e^{-t} d t$ 
represents the incomplete $\Gamma$-function. The derivative at the wall: $\theta^{\prime}(0)=-\frac{\sqrt[3]{3}}{\Gamma\left(\frac{1}{3}\right)}$, gives the local the Nusselt as:

$$
N u_{R}=\frac{\sqrt[3]{3}}{\Gamma\left(\frac{1}{3}\right)}\left(2 \bar{U}_{\max } \operatorname{Pr} R e\right)^{1 / 3} \bar{x}^{-1 / 3}
$$

The heat equation Eq. 4 integrated across the pipe will be useful (see the integral equation 12 thereafter) in his global form :

$$
\frac{\partial}{\partial \bar{x}} \int_{0}^{1}(\bar{r} \bar{u} \bar{T}) d \bar{r}=\left.\frac{\partial}{\partial \bar{r}} \hat{T}\right|_{\bar{r}=1} .
$$

We define a Poiseuille averaged temperature to be

$$
\bar{T}_{m}=4 \int_{0}^{1}\left(1-\bar{r}^{2}\right) \bar{T} d \bar{r}
$$

in $\bar{x}=0$, at the end of the collector, the value of the convective flux is computed, its value will then remain constant for $\bar{x}>0$. The equation for the mean temperature becomes

$$
\bar{T}_{m}=4 \varepsilon^{2} \int_{0}^{\infty} 2 \hat{r} T(x, \hat{r}) d \hat{r}
$$

Since $\eta=\hat{r} / \ell^{1 / 3}$ we have $\bar{T}_{m}=8 \varepsilon^{2} \bar{\ell}^{2 / 3} \int_{0}^{\infty} \eta \theta d \eta$ and as $\int_{0}^{\infty} \eta \theta d \eta=\frac{3 \sqrt[3]{3}}{2 \Gamma\left(\frac{1}{3}\right)}$ the final expression of the prediction of the mean temperature in the Lévêque description:

$$
\bar{T}_{m}=\varepsilon^{2} \bar{\ell}^{2 / 3} \frac{12 \sqrt[3]{3}}{\Gamma\left(\frac{1}{3}\right)}
$$

Figure 7 shows on the left an example of pure Lévêque problem (a Poiseuille flow is in the tube, i.e. we removed the buoyant term during the resolution and imposed a pressure gradient) and on the right, we plot the temperature profiles for an effective case $(R e=5000)$. We see that the Lévêque solution is a good first approximation of the temperature profiles in the collector part.

\subsubsection{The modified Graetz problem}

Another useful well known solution of heat equation in pipes is the Graetz problem (29]). It corresponds to a change in temperature boundary condition in a Poiseuille flow at large Péclet number. The heat equation in a Poiseuille flow is

$$
\bar{U}_{p} \frac{\partial \bar{T}}{\partial \bar{x}}=\frac{1}{R e P r}\left(\frac{1}{\bar{r}} \frac{\partial}{\partial \bar{r}}\left(\bar{r} \frac{\partial \bar{T}}{\partial \bar{r}}\right)+\frac{\partial^{2} \bar{T}}{\partial \bar{x}^{2}}\right),
$$

we define $\tilde{x}=\operatorname{Re} \operatorname{Pr} \bar{x}$, and $\tilde{r}=\bar{r}$ so that the the heat equation is asymptotically, at large $R e$ :

$$
\tilde{U}_{p} \frac{\partial \tilde{T}}{\partial \tilde{x}}=\frac{1}{\tilde{r}} \frac{\partial}{\partial \tilde{r}}\left(\tilde{r} \frac{\partial \tilde{T}}{\partial \tilde{r}}\right), \text { with } \tilde{U}_{p}=\left(1-\tilde{r}^{2}\right),
$$

with, here, boundary conditions $\tilde{T}(\tilde{x}=0, \tilde{r})$ given and $\partial \tilde{T}(\tilde{x}>0,1) / \partial \tilde{r}=0$. The temperature at the center of the chimney is plotted as a function of $\tilde{x}$ on figure 8 . It is clear that the Graetz asymptotic description is pertinent in the chimney even if buoyancy is present because the solution is to a good approximation a function of $\tilde{x}=\bar{x} / R e$. 


\subsection{One dimensional simple analysis}

\subsubsection{With exchange coefficient}

Before looking at more elaborate asymptotic models than the Lévêque and Graetz ones, the most simple way to analyse the flow is the one dimensional approximation (in the case of solar tower, there is a large literature on that [16. 17, 33...). In the tower, taking into account the fact that the tower is elongated $\left(L_{c} \gg R\right)$ allows that $1 \mathrm{D}$ equations may be settled. Hence, the equations (14) are integrated across the constant section, of surface $S=\pi R^{2}$. The equations are written with dimensions. The mass conservation Eq. 1 is

$$
\frac{\partial}{\partial x}(S U)=0
$$

The momentum conservation Eq. 2/3 gives all over the tube for $-\ell<x<L_{c}$ :

$$
\rho_{0} \frac{\partial}{\partial x}\left(S U^{2}\right)=-S \frac{\partial}{\partial x} p+\rho_{0} g \alpha\left(T-T_{0}\right) S-\frac{c_{f} S}{2 R}\left(\frac{\rho_{0} U^{2}}{2}\right)
$$

the heat equation Eq. 4 is then in two parts, for $-\ell<x<0$ :

$$
c_{p} \frac{\partial}{\partial x}\left(\rho_{0} S U T\right)=-h 2 \pi R\left(T-T_{w}\right) \text { and for } x>0: c_{p} \frac{\partial}{\partial x}\left(\rho_{0} S U T\right)=0 .
$$

Note that $\rho_{0}$ is constant, and that the problem is steady $\frac{\partial}{\partial t}=0$. Further more $S$ is constant. Those equation were written using $U=\left(2 \int_{0}^{R} u r d r\right) / R^{2}$ the mean velocity and $T$ the mean temperature, a shape coefficient:

$$
R^{2}\left(2 \int_{0}^{R} u^{2} r d r\right) /\left(2 \int_{0}^{R} u r d r\right)^{2}
$$

should be introduced. The term with $h$ is the exchange coefficient in the collector. The 10 11 system is different from the one presented in [17, 16, 33. as here we are in a steady incompressible flow. As we are here in laminar flow $c_{f}=64 /(2 U R / \nu)$, and the viscous term in Eq. 11 is only $-8 \mu U S / R^{2}$ (adaptation to turbulent flow are just done in changing this friction coefficient).

From Eq. 10, the velocity is $U$ is constant in the whole pipe. The heat equation Eq. 12 is solved, the mean temperature rises in the collector and is constant in the chimney. With $\lambda=\frac{2 \pi R h}{\rho_{0} c_{p} S U}$, the solution clearly involves an exponential $e^{-\frac{2 \pi R h}{\rho_{0} c_{p} S U} x}$, this is:

$$
\left\{\begin{array}{l}
T(x)=T_{0}+\left(T_{w}-T_{0}\right)\left(1-\exp \left(-\frac{x+\ell}{\lambda}\right)\right), \text { for }-\ell<x<0 \\
T(x)=T_{0}+\left(T_{w}-T_{0}\right)\left(1-\exp \left(-\frac{\ell}{\lambda}\right)\right), \text { for } 0<x<L_{c}
\end{array}\right.
$$

as the temperature is known, one solves the pressure from momentum equation, which reduces to the pressure gradient, Boussinesq term and viscous term (the inertia in Eq. 11 is zero as $U$ is constant). Starting from 0 (and as we defined $\left.U_{0}=\left(R^{2} \rho_{0} g \alpha\left(T_{w}-T_{0}\right)\right) / \mu\right)$, then in $x=0$, the pressure is continuous, and:

$$
\left\{\begin{aligned}
R e \frac{p}{\rho_{0} U_{0}^{2}} & =-\frac{\lambda}{R}\left(1-\exp \left(-\frac{x+\ell}{\lambda}\right)\right)+\frac{(x+\ell)}{R}\left(1-8 \frac{U}{U_{0}}\right), \text { for }-\ell<x<0 \\
\operatorname{Re} \frac{p}{\rho_{0} U_{0}^{2}} & =-\frac{x}{R} e^{-\frac{\ell}{\lambda}}+\frac{\lambda}{R}\left(e^{-\frac{\ell}{\lambda}}-1\right)+\frac{(x+\ell)}{R}\left(1-8 \frac{U}{U_{0}}\right), \text { for } 0<x<L_{c} .
\end{aligned}\right.
$$


As the pressure must be zero in $x=L_{c}$, this allows to find, if $\lambda$ is constant, the value of the mean velocity $\bar{U}=U / U_{0}$ :

$$
\bar{U}=\frac{e^{-\frac{\ell}{\lambda}}\left(e^{\ell / \lambda}\left(\ell-\lambda+L_{c}\right)+\lambda-L_{c}\right)}{8\left(\ell+L_{c}\right)} .
$$

If the heat transfer coefficient is not constant then we have to solve numerically by iteration this equation.

But, from our numerical tests, this exponential behavior is not very good to describe the temperature. We think that this kind of model is interesting as it gives the salient mechanisms but one has to adjust the heat exchange factor $h$ to fit the numerical simulations. Furthermore, as in this model, there is absolutely no inertia, and the flow is constant, we can not reproduce the hump observed in figure 5 and of course we can not reproduce the maximum of pressure observed on this same figure.

So, we have to add some 2D effects to go further. The most simple thing to do, is to exploit the results from the Lévêque heating.

\subsubsection{With the help of Lévêque and Graetz}

We change a bit the previous mono dimensional description because the heat equation with an exchange factor is too crude. We suppose always that the flow is a Poiseuille one, of mean value $\bar{U}$. As this flux is constant, inertia does not change in the momentum equation $11 . \partial_{\bar{x}} \bar{U}^{2}=0$. First in the heated part, neglecting the buoyancy term in Eq. 11, we have only the pressure gradient and the viscous term. Then, momentum equation 11 reduces without dimensions (with $\bar{p}(0)$ value of the pressure in 0 , and $(\bar{p}(0)-0) / \bar{\ell}$ the pressure gradient) to:

$$
0=\frac{-\bar{p}(0)}{\bar{\ell}}-\frac{8}{R e} \bar{U} .
$$

The heat equation reduces to the Lévêque problem so that in $\bar{x}=0$, the mean temperature is the same than equation 7 which gives equation 9 . Second in the adiabatic part, this buoyancy term: $\bar{T}_{m}=4.06\left(\bar{U}_{\max } P e\right)^{-2 / 3} \bar{\ell}^{2 / 3}$ is now the driving term in the momentum equation 11 , the pressure gradient is now $(0-p(0)) / L_{c}$, so the momentum equation in the chimney is:

$$
0=\frac{\bar{p}(0)}{L_{c}}-\frac{8}{R e} \bar{U}+\frac{1}{R e} \bar{T}_{m}
$$

this gives the mean value of the velocity

$$
\bar{U}=\frac{\bar{T}_{m}}{8\left(1+\ell / L_{c}\right)} .
$$

This equation is close to Eq. 13, the difference is that here we used here a better approximation for the mean temperature in using directly the Lévêque solution rather solving Eq. 12 with exponentials. 


\subsubsection{Final velocity prediction}

From this analysis, one obtains (the max value is twice the mean value) with dimensions:

$$
U_{\max }=1.05 \frac{\nu}{R} \frac{(P r)^{-2 / 5} G r^{3 / 5} \ell^{2 / 5}}{\left(1+\ell / L_{c}\right)^{3 / 5}} \quad \text { with } R e=G r=\frac{g \alpha \Delta T R^{3}}{\nu^{2}}
$$

As expected if $\Delta T$ increases, $U_{m}^{*}$ increases, as viscosity $\nu$ decreases $U_{\max }^{*}$ increases $\left(\nu^{-1 / 5}\right)$ As $\ell$ increases $U_{\text {max }}^{*}$ increases (of course $\ell<L_{c}$, if not $U_{\max }^{*}$ decreases with $\ell$ ). This gives a reasonable approximation of the velocity see figure 9

With some 2D ingredients, the $1 \mathrm{D}$ model is now better, but we can add more $2 \mathrm{D}$ effects to improve the description.

\subsection{Two dimensional Asymptotic analysis; RNSP equa- tions}

In this part we construct an asymptotic model $(R e \gg 1)$ for the flow which takes into account the Lévêque and the modified Graetz problem. Further more this asymptotic model takes into account the balance of buoyancy, inertia and viscous dissipation in the momentum. This is in fact a kind of boundary layer problem with the axi symmetrical long wave equations (or Reduced Navier Stokes Prandtl problem, RNSP, as we have Prandtl equations or boundary layer equations, see 2] 19, 21] for comparisons with Navier Stokes). These model equations are very similar to those of Aung et al. 1, they use the same boundary conditions in pressure at entrance and exit. We use the Graetz scales: longitudinaly $R e^{-1} R$ and transversaly $R$ :

$$
\tilde{x}=R e^{-1} \bar{x}=\frac{x}{R R e}, \tilde{r}=\bar{r}=\frac{r}{R}
$$

and the same for the velocity

$$
\tilde{u}=\bar{u}=\frac{u}{U_{0}}, \tilde{v}=R e^{-1} \bar{v}=\frac{v}{U_{0} R e},
$$

and temperature and pression remain unchanged in scales:

$$
T=T_{0}+\Delta T \tilde{T}, \tilde{p}=\frac{p-p_{0}}{\rho_{0} U_{0}^{2}}
$$

With this change of scale, as $R e \gg 1$, the incompressibility is unchanged:

$$
\frac{\partial \tilde{u}}{\partial \tilde{x}}+\frac{1}{\tilde{r}} \frac{\partial \tilde{r} \tilde{v}}{\partial \tilde{r}}=0
$$

But, now, pressure is constant across the tube at this scale:

$$
0=-\frac{\partial \tilde{p}}{\partial \tilde{r}}
$$

Longitudinal momentum is a balance between inertia, pressure, transverse viscous term and buoyancy:

$$
\tilde{u} \frac{\partial \tilde{u}}{\partial \tilde{x}}+\tilde{v} \frac{\partial \tilde{u}}{\partial \tilde{r}}=-\frac{\partial \tilde{p}}{\partial \tilde{x}}+\frac{1}{\tilde{r}} \frac{\partial}{\partial \tilde{r}}\left(\tilde{r} \frac{\partial \tilde{u}}{\partial \tilde{r}}\right)+\tilde{T} .
$$


The heat equation is:

$$
\tilde{u} \frac{\partial \tilde{T}}{\partial \tilde{x}}+\tilde{v} \frac{\partial \tilde{T}}{\partial \tilde{r}}=\frac{1}{\operatorname{Pr}}\left(\frac{1}{\tilde{r}} \frac{\partial}{\partial \tilde{r}}\left(\tilde{r} \frac{\partial \tilde{T}}{\partial \tilde{r}}\right)\right)
$$

Boundary conditions are no slip at the wall $\tilde{u}(\tilde{x}, \tilde{r}=1)=0, \tilde{v}(\tilde{x}, \tilde{r}=1)=0$, prescribed temperature at the collector wall $\tilde{T}(-\tilde{\ell}<\tilde{x}<0, \tilde{r}=1)=1$, at the entrance $\tilde{T}(\tilde{x}=-\tilde{\ell}, \tilde{r})=0$ and no flux in the adiabatic chimney $\partial \tilde{T} / \partial \tilde{r}=0$ in $\tilde{x}>0$ and $\tilde{r}=1$. The chosen configuration is with $\tilde{p}=0$ at the entrance $\tilde{x}=-\tilde{\ell}$ for any $\tilde{r}$, and at the output $\tilde{x}=\tilde{L}_{c}$ the pressure is zero again: $\tilde{p}=0$ (for any $\tilde{r})$.

The equations are solved in finite differences, implicit discretisation in $\tilde{x}$ and with iteration at each $\tilde{x}$ to obtain the pressure gradient for which the velocity $\tilde{v}$ is zero at the wall (see 2] and 23] for details). In fact those equations (from Eq. 14 to 17) are parabolic in space (we solve them by marching in space $\tilde{x}$ ), so the output condition can not be imposed. To solve the problem with the relevant output, iterations of the whole marching scheme are done in order to obtain this output pressure equals to zero: for a given $\bar{U}_{\max }$ (at the entrance $\tilde{u}=\bar{U}_{\max }\left(1-\tilde{r}^{2}\right), \tilde{v}=0$ and $\left.\bar{T}(\bar{x}=-\bar{\ell}, \bar{r})=0\right)$, we adjust $\bar{U}_{\max }$ to obtain the good exit value $\tilde{p}\left(\tilde{L}_{c}, \tilde{r}\right)=0$.

Note that in the paper [1, they impose the same boundary conditions, but they do just a one shot computation: starting from a value of the entrance flux and a zero pressure, they do the computation up to the position were the pressure becomes zero again. So the length of the tube is not a constant when they vary the flux. Here the length is constant, so we have to iterate to find the right flux.

Finally, the system of Eq 14 17 contains the Poiseuille flow solution, the Lévêque heat problem and the Graetz heat problem itself, furthermore, it contains the pioneering Elenbass system of equations 7 .

Results of this Reduced Navier Stokes Prandtl model will be discussed in coordination with the next asymptotic model which is a Linearized Navier Stokes model. Note at this point that this model is not so far from the 1D model: integrating the RNSP across the section gives the 1D model. In fact integrating 14 over the section gives exactly the global equation 10 . The equation 15 corresponds to a pressure depending on $\bar{x}$ only. Integrating Eq. 16 over the section gives the global equation of momentum 11 and finally integrating the heat equation 17 gives the integral counter part Eq. 12 . But the RNSP system contains more information than the $1 \mathrm{D}$ model, as the radial quantities are computed with a better description.

\subsection{Two dimensional perturbative analysis: LNS Linearized Navier Stokes}

Another final point of view is proposed here, it is supposed that the flow remains mainly a Poiseuille one (which was supported by NS results). The temperature change induces only a small perturbation so that the basic flow is Poiseuille (given by $\bar{U}_{p}=\bar{U}_{\max }\left(1-\bar{r}^{2}\right)$ and for pressure $\bar{p}_{p}=-4(\bar{x}+\bar{\ell}) \bar{U}_{\text {max }} / R e$ ). Hence the Navier Stokes equations are linearised around the basic Poiseuille flow with 
temperature 0 . The linearised heat equation 4 is then:

$$
\bar{r} \bar{U}_{p} \frac{\partial \bar{T}_{1}}{\partial \bar{x}}=\frac{1}{R e P r}\left(\frac{\partial}{\partial \bar{r}}\left(\bar{r} \frac{\partial \bar{T}_{1}}{\partial \bar{r}}\right)+\frac{\partial}{\partial \bar{x}}\left(\bar{r} \frac{\partial \bar{T}_{1}}{\partial \bar{x}}\right)\right)
$$

it gives the field of perturbation for temperature $\bar{T}_{1}$. Notice that the longitudinal term $\left(\partial_{\bar{x}}^{2} \bar{T}_{1}\right)$ is not dropped here. Then, we look at a perturbation field $\bar{u}_{1}$, $\bar{v}_{1}$ induced by this temperature $\bar{T}_{1}$, the linearised Navier Stokes Boussinesq equations 1,3 are then (again with all the $\partial_{\bar{x}}^{2}$ terms):

$$
\begin{gathered}
\frac{\partial \bar{r} \bar{v}_{1}}{\partial \bar{r}}+\frac{\partial \bar{r} \bar{u}_{1}}{\partial \bar{x}}=0 \\
\bar{r} \bar{U}_{p} \frac{\partial \bar{v}_{1}}{\partial \bar{x}}=-\bar{r} \frac{\partial \bar{p}_{1}}{\partial \bar{r}}+\frac{1}{R e}\left(\frac{\partial}{\partial \bar{r}}\left(\bar{r} \frac{\partial \bar{v}_{1}}{\partial \bar{r}}\right)+\frac{\partial}{\partial \bar{x}}\left(\bar{r} \frac{\partial \bar{v}_{1}}{\partial \bar{x}}\right)\right) \\
\bar{r} \bar{U}_{p} \frac{d \bar{u}_{1}}{d \bar{x}}+\bar{r}_{1} \frac{d \bar{U}_{p}}{d \bar{r}}=-\bar{r} \frac{\partial \bar{p}_{1}}{\partial \bar{x}}+\frac{1}{R e}\left(\frac{\partial}{\partial \bar{r}}\left(\bar{r} \frac{\partial \bar{u}_{1}}{\partial \bar{r}}\right)+\frac{\partial}{\partial \bar{x}}\left(\bar{r} \frac{\partial \bar{u}_{1}}{\partial \bar{x}}\right)\right)+\frac{r}{R e} \bar{T}_{1}
\end{gathered}
$$

Boundary conditions for the velocities $\bar{u}_{1}, \bar{v}_{1}$ are no slip velocity at the walls and Neumann at the input and output. The perturbed pressure is imposed to be zero $\bar{p}_{1}=0$ at the exit but Neumann at the entrance.

The value of the max of the velocity, $\bar{U}_{\max }$ is ajusted so that the pressure must be zero at the entrance:

$$
\bar{p}=-4(\bar{x}+\bar{\ell}) \bar{U}_{\max } / R e+\left(\bar{p}_{1}(\bar{x}, 0)-\bar{p}_{1}(-\bar{\ell}, 0)\right) / R e,
$$

this is the sum of the Poiseuille contribution $\bar{p}_{p}=-4(\bar{x}+\bar{\ell}) \bar{U}_{\max } / R e$ plus the $\bar{p}_{1} / R e$ from the linearized problem. Of course, that is at this point, when we recompose $\bar{p}_{p}$ and the perturbation $\bar{p}_{1}$, that we do an approximation which is that the perturbation field is as great as the basic one. Of course, this invalidates the whole process, but we use anyway this approximation as a first step. This decomposition in a Stokes problem plus another one is completely reminiscent to the superposition method developed by Le Quéré [18] (and [13]). On any solution one can superpose a linear combination of a Poiseuille flow requiring that the total pressure drop between inlet and outlet is equal to zero (see 18]).

Those equations 19,21 are elliptic. They are written in variational form and solved with finite elements with freefem++ [8]. We present next the results of this NS linear model compared with the RNSP model.

\subsection{Some comparisons of the asymptotic models}

About the 1D model, in the first figures 4, 5 and 6, the pressure has been plotted with the $1 \mathrm{D}$ prediction. We see that it works just for the entrance, but the pressure $\bar{p}(0)$ is always far lower in the $1 \mathrm{D}$ model than in the NS computations. Nevertheless, the scale of pressure $(1 / R e)$ is the good one, which is a information in itself. The previous two models 3.3 and 3.4 have been compared in order to reproduce the flow in the tube. First, for moderate value of $R e(200$ figure 10, the overall pressure distribution is well reproduced. The decreasing/ increasing pressure is observed, this corresponds to the $1 \mathrm{D}$ model. This corresponds to the fact that the Poiseuille flow is not very perturbed and the viscous effects are important so that equilibrium is attained at small distances (inertia is negligible).

Increasing the Reynolds to 750 allows to reproduce the hump in the pressure (figure 11. This effect is clearly an inertial effect due to acceleration induced by 
the heating: near the wall the velocity increases, by mass conservation velocity decreases on the axis. This decrease of velocity in the core flow is associated to a small increase of pressure. The adiabatic part promotes a decrease of the wall velocity, so an increase of core flow velocity, hence an increase of pressure (note that some Triple Deck structure can be introduced here like in [20]). That this effect which creates the hump of pressure observed on the curves. In increasing more the Reynolds, we can reproduce the behavior of figure 6 with the two asymptotic models. This effect is displayed on figure 12 it shows that both asymptotic models reproduce the positive extremum of pressure at the junction collector/ chimney. Unfortunately, the comparison with the full Navier Stokes of figure 6 is only qualitative. Reasons for discrepancy are not clear as the differences between RNSP and Linearised NS are small.

From these comparisons, we see that the flow remains close to a Poiseuille (introduced as hypothesis in Linearised NS). We see that Linearised Navier Stokes is close to Reduced Navier Stokes, this shows that longitudinal terms $\partial_{\bar{x}}^{2}$ are negligible. This shows that the hypothesis of linearisation is relevant. About the one dimensional model, the problem is that the entrance length of temperature is not very well estimated, a reason is that there is no inertia in the momentum. Another is that inertia in the heat equation is crude: the exponential behavior of the temperature due to the exchange coefficient is not the proper one. Using a mix with Lévêque solution as in paragraph 3.2 .2 is a better way to model the heat equation, but not enough to have an enough detailed description of the flow. The main reason is of course that the 1D model is too much constrained by the choice of velocity profile.

\section{Discussion and conclusion}

As stated in the introduction, this is a model configuration. So, we tried to construct a consistent hierarchy of incompressible models (from 1D to Navier Stokes Boussinesq axi, via asymptotic models). We consider flows in a straight long (length is from 10 to 100 in units of the radius) tube heated at the base (on a distance of one to five radius of the pipe). Free convection starts the flow, but aspiration of fresh fluid creates finally a flow dominated in the lower heated part by forced convection and in the second adiabatic part by free convection. For those geometries, if the Reynolds (or in fact the Grashof, or in practice the difference of temperature) is large enough, the final steady flow is very close to a Poiseuille flow, the amplitude is function or the geometry and of the Grashof. The pressure decreases in the heated part of the tube and increases in the adiabatic part, the order of magnitude of the pressure is one over Grashof. This may be explained with 1D theory. But, if the Grashof (or Reynolds, they are the same) increases, the pressure variation along the tube experiences a change. Due to the wall heating and mass conservation, the core flow is decelerated and accelerated: the pressure increases a bit and then decreases. This hump in pressure obtained in full numerical simulations, is only explained by the proposed asymptotic models, not by the 1D one. If the Grashof is large enough, a positive maximum of pressure may appear in the tube near $\bar{x}=0$. This global numerical behavior has been reproduced fairly well with the two asymptotic models. The first one is in the boundary layer spirit (Prandtl equations are written in the whole tube), this first model is consistent with Graetz and Lévêque scales. 
This model is parabolic in space, ellipticity is recovered by the relaxation on the mean velocity in order to match the boundary condition in pressure at both ends. The second model is elliptic in space, it is a linearisation of the Navier Stokes equations due to the supposed small change in temperature. Both are mixed convection problems as we start form a value of the entrance flux and we need iteration to meet zero pressure at exit. This iteration breaks the apparent parabolic in space resolution of RNSP model by a global interaction. The one dimensional model is consistent with the RNSP as it corresponds to its average across the section (but too many details are removed due to the averaging process).

We took a zero pressure drop in the tube, this hypothesis may be discussed now a bit more. One may add an accelerating pressure drop as in [6] or in [15. This entrance pressure drop corresponds to the history of the acceleration of the flow, recent computations question this hypothesis [12. For our asymptotic models, changing the pressure at the entrance is not a real problem. Hence, the methods described here may be applied to the benchmark [5] and 6. Notice that in [15], a discussion of the influence of the boundary conditions in a vertical channel with heated walls is proposed. We should as well apply our methods to this configuration. The real question is how to model the exterior of the tower, and whether down and top couplings are possible. This question of the influence of the surrounding flow is an open question which has no real responses in the literature now. But very recently Garnier et al. [10, 11] have done a very interesting first step in this direction. They did a systematic computation of a heated flow in a 2D channel (in a different configuration, the one like [15, 13]), this channel of finite given length being at the center of a larger box. The size of the box has been increased and increased in order to simulate an infinite domain. From the results, it seems (9) that the flow approaches roughly the zero difference of pressure, a better model would be a Robin condition, were $L_{i n}$ is observed to be fonction of $R e$ in the simulations, like

$$
p+L_{i n} \frac{\partial p}{\partial x}=0
$$

Again, a real solar tower is far from this model (entrance effects, small out put effects, conduction in the walls, radiation, unsteady effects, effect of the outside, turbulence...), but we have seen that even a simple geometry can produce unexpected distributions of pressure. Furthermore, the coupling with the external flow will be very important in practical configurations. We then think that one dimensional models are not enough to study real devices, nevertheless boundary layer approximations such as those proposed may be written in turbulent form ([3] ), this may be a good starting point to analyze the flow. The proposed asymptotic models (Linearised NS and RNSP) show very good qualitative agreement with full Navier Stokes, so that, between full Navier Stokes resolution and over simplified $1 \mathrm{D}$ theories there exists still place for some asymptotic models of boundary layer type which provide some useful informations on the flow.

Thanks The authors wish to thank ENSTA Paristech (aka. Techniques Avancées) to provide links between them trough a series of courses at ENIT Tunis. 


\section{References}

[1] W. Aung, L. Fletcher, and V. Sernas, "Developing laminar free convection between vertical flat plates asymmetric heating". International Journal of Heat and Mass Transfer, 15:2293 -2307, 1972.

[2] Franz Chouly \& P.-Y. Lagrée (2012): "Comparison of computations of asymptotic flow models in a constricted channel.", Applied Mathematical Modelling 36 (2012), pp. 6061-6071doi

[3] T. Cebeci J. Cousteix (2005) Modeling and Computation of Boundary-Layer Flows Second Edition, Springer, 502 pages

[4] Chitsomboon, T. (2001). A validated analytical model for flow in solar chimney. International Journal of Renewable Energy Engineering 3 (2), 339-346.

[5] G. Desrayaud, R. Bennacer, J.P. Caltagirone, E. Chenier, A. Joulin, N. Laaroussil, et K. Motjtabi. (2007) Etude numérique comparative des écoulements thermoconvectifs dans un canal vertical chauffé asymmétriquement. In 8 ème Colloque Inter-Universitaire Franco-Québécois sur la Thermique des Systèmes, CIFQ2007/ART-06-14, 28-30 (Mai 2007).

[6] G. Desrayaud, E. Chénier, A. Joulin, A. Bastide, B. Brangeon, J.P. Caltagirone, Y. Cherif, R. Eymard, C. Garnier, S. Giroux-Julien, Y. Harnane, P. Joubert, N. Laaroussi, S. Lassue, P. Le Quéré, R. Li, D. Saury, A. Sergent, S. Xin, A. Zoubir (2013) "Benchmark solutions for natural convection flows in vertical channels submitted to different open boundary conditions" International Journal of Thermal Sciences, Volume 72, October 2013, pp. 18?33, 2013.

[7] W. Elenbaas "Heat dissipation of parallel plates by free convection" Physica Volume 9, Issue 1, January 1942, Pages 1-28

[8] http://www.freefem.org/ff++/

[9] C. Garnier (2014) "Modélisation numérique des écoulements ouverts de convection naturelle au sein d'un canal vertical asymétriquement chauffé" $\mathrm{PhD}$ Thesis University Paris-Sud Orsay

[10] Garnier C., Sergent A., Fraigneau Y. Le Quéré "Comparative study of numerical simulations of a $2 \mathrm{~d}$ buoyancy-driven flow in a vertical channel asymmetrically heated with or without external domain", Proceedings of the 15th International Heat Transfer Conference, 2014, Kyoto, Japan IHTC15-9291

[11] Garnier C., Sergent A., Fraigneau Y. Le Quéré Etude numérique de l'interaction entre un canal vertical asymétriquement chauffé et son environnement extérieur

[12] C. Garnier A. Sergent, P. Le Quéré, Private communication.

[13] C. Garnier A. Sergent, P. Le Quéré (2013), "Modélisation et influence des conditions limites pour des écoulements $2 \mathrm{~d}$ semi-confinés" XIème Colloque Interuniversitaire Franco-Québécois sur la Thermique des Systèmes 3-5 juin 2013, Reims 
[14] Gerris Flow Solver http://gfs.sourceforge.net/wiki/index.php/Main_Page

[15] Hua Sun, Ru Li, Eric Chénier, Guy Lauriat (2011) "On the modeling of aiding mixed convection in vertical channels" Heat Mass Transfer 48, 7 (2012) 1125-1134" DOI : 10.1007/s00231-011-0964-8

[16] A. Koonsrisuk, S. Lorente, A. Bejan (2010) "Constructal solar chimney configuration" International Journal of Heat and Mass Transfer 53 (2010) $327-333$

[17] Koonsrisuk,A.,(2012) Mathematical modeling of sloped solar chimney power plants Energy $46582-589$

[18] P. Le Quéré (2008) "On the computation of some external or partially enclosed natural convection flows", The 19th International Symposium on Transport Phenomena, 17-20 August, 2008, Reykjavik, I

[19] P.-Y. Lagrée \& S. Lorthois (2005): "The RNS/Prandtl equations and their link with other asymptotic descriptions. Application to the computation of the maximum value of the Wall Shear Stress in a pipe", Int. J. Eng Sci., Vol 43/3-4 pp 352-378.

[20] P.-Y. Lagrée (1999): "Thermal mixed convection induced locally by a step in surface temperature in a Poiseuille Flow in the framework of Triple Deck", International Journal of Heat and Mass Transfer, Vol 42, pp. 2509-2524.

[21] P.-Y. Lagrée E. Berger, M. Deverge, C. Vilain \& A. Hirschberg (2005): "Characterization of the pressure drop in a 2D symmetrical pipe: some asymptotical, numerical and experimental comparisons", ZAMM: Z. Angew. Math. Mech. 85, No. 2, pp. 141-146.

[22] P.-Y. Lagrée (2001): "Removing the marching breakdown of the boundary layer equations for mixed convection above a horizontal plate", International Journal of Heat and Mass Transfer, Vol 44/17, pp. 3359-3372.

[23] Lagrée P.-Y. (2010) Interactive Boundary Layers In: Asymptotic Methods in Fluid Mechanics: Survey and Recent Advances, CISM Courses and Lectures, vol. 523", H. Steinrück (ed.); Springer-Verlag, Wien New York, 2010, ISBN: 978-3-7091-0407-1, pp. 247-286

[24] P.-Y. Lagrée L. Staron S. Popinet (2011): "The granular column collapse as a continuum: validity of a Navier-Stokes model with a $\mu(I)$-rheology" Journal of Fluid Mechanics, pp1-31 , doi:10.1017/jfm.2011.335

[25] A. Leontiev (1985) "Théorie des échanges de chaleur et de masse", ed. MIR.

[26] Lévêque, A.: Les lois de la transmission de chaleur par convection. Ann. des Mines. XIII (1928) S. 201-299, 305- 362 381-415.

[27] T. J. Pedley, "The Fluid Mechanics of Large Blood Vessels", Cambridge University Press, 1980, 633p

[28] S. Popinet "Gerris: a tree-based adaptive solver for the incompressible Euler equations in complex geometries" J. Comput. Phys. 190(2):572-600, 2003. 
[29] L. Prandtl (1952)"Guide à travers la mécanique des fluides", Dunod 1952

[30] M. Padki and S. A. Sherif (1999) on a simple analytical model for solar chimneys . Int. J. Energy Res., 23, 345349 (1999)

[31] Pastohr H, Kornadt O, Gurlebeck K. (2004) "Numerical and analytical calculations of the temperature and flow field in the upwind power plant." Int J Energy Res 2004;28:495-510.

[32] Roozbeh Sangi, Majid Amidpour, Behzad Hosseinizadeh (2011) Modeling and numerical simulation of solar chimney power plants Solar Energy 85 (2011) 829-838

[33] Tingzhen, M., Wei, L., Guoliang, X., (2006). Analytical and numerical investigation of the solar chimney power plant systems. International Journal of Energy Research, 30:861-873.

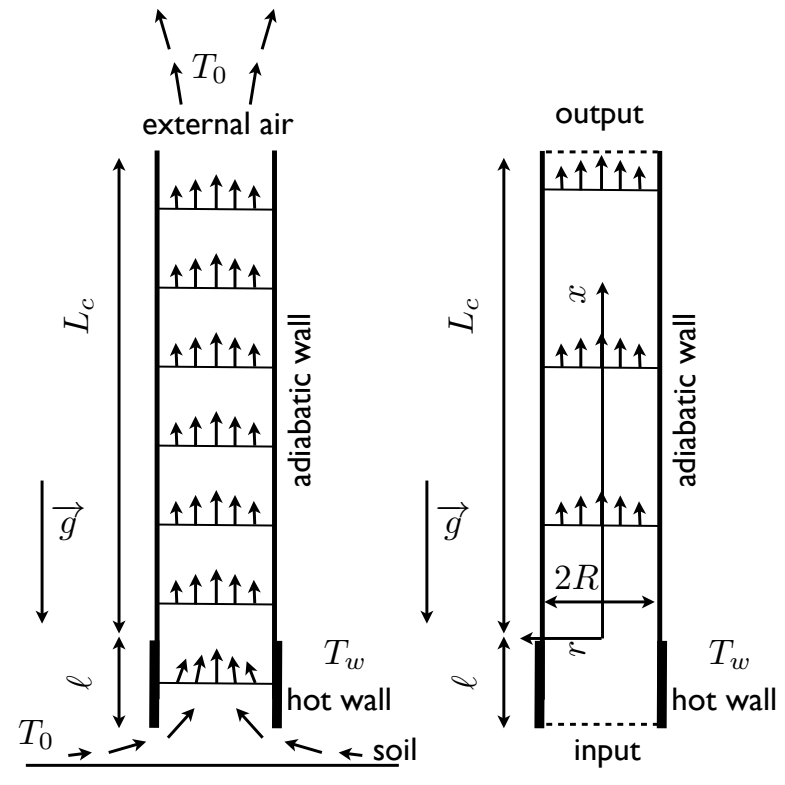

Figure 1: Left, an over simplified model for a solar tower, air heated at the base is aspirated and flows in the tower; at the output of the chimney, a buoyant jet develops. A real solar tower is far more complicated, the collector (heated part) is horizontal, and the soil is heated it self as well. Right, the numerical model is a straight pipe (a cylinder). We impose $p=0$ at the entrance and Neumann conditions for $u\left(\partial_{x} u=0, v=0\right)$ at the input $x=-\ell$, Dirichlet no slip condition on the tube $\left(u=v=0, r=R,-\ell<x<L_{c}\right)$ and at the output $x=L_{c}$ the pressure is zero again: $p=0$ and velocity is $\partial_{x} u=0, v=0$. The heated part $\left(T(-\ell<x<0, r=R)=T_{w}\right)$ is called the collector, the adiabatic one $\left(\partial_{r} T\left(0<x<L_{c}, r=R\right)=0\right)$, the chimney; $T=T_{0}$ at the entrance, $\partial_{x} T=0$ at the exit, There is no radiation and no conduction in the wall in this model. 


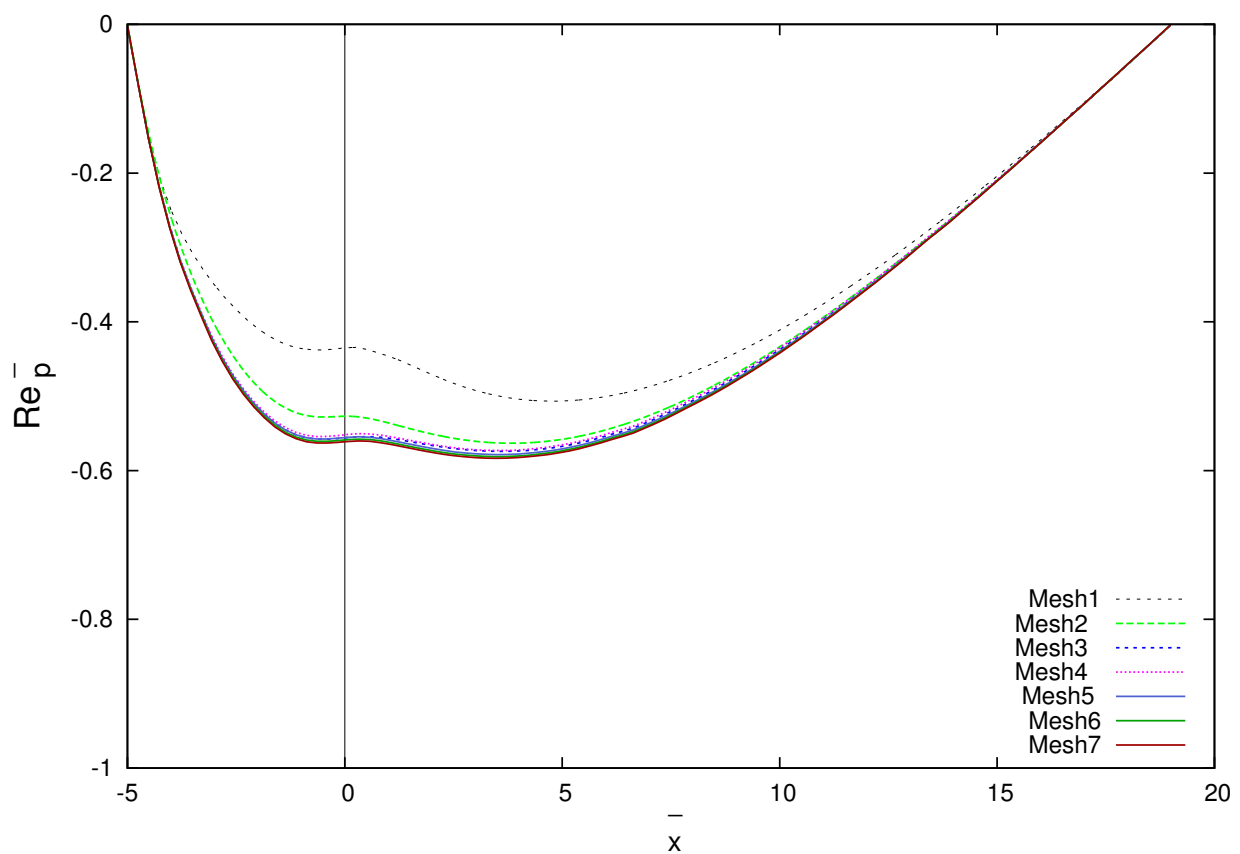

Figure 2: Pressure times Reynolds $\bar{p}(\bar{x}, \bar{r}=0)$ Re along the axis of the pipe with a collector of length $\bar{\ell}=5, \bar{L}_{c}=19$ (color online), for Reynolds numbers (Re= 800 ) and for various mesh refinement, mesh $N-1$ stands for $\Delta \bar{x}=\Delta \bar{r}=2^{-N}$ 


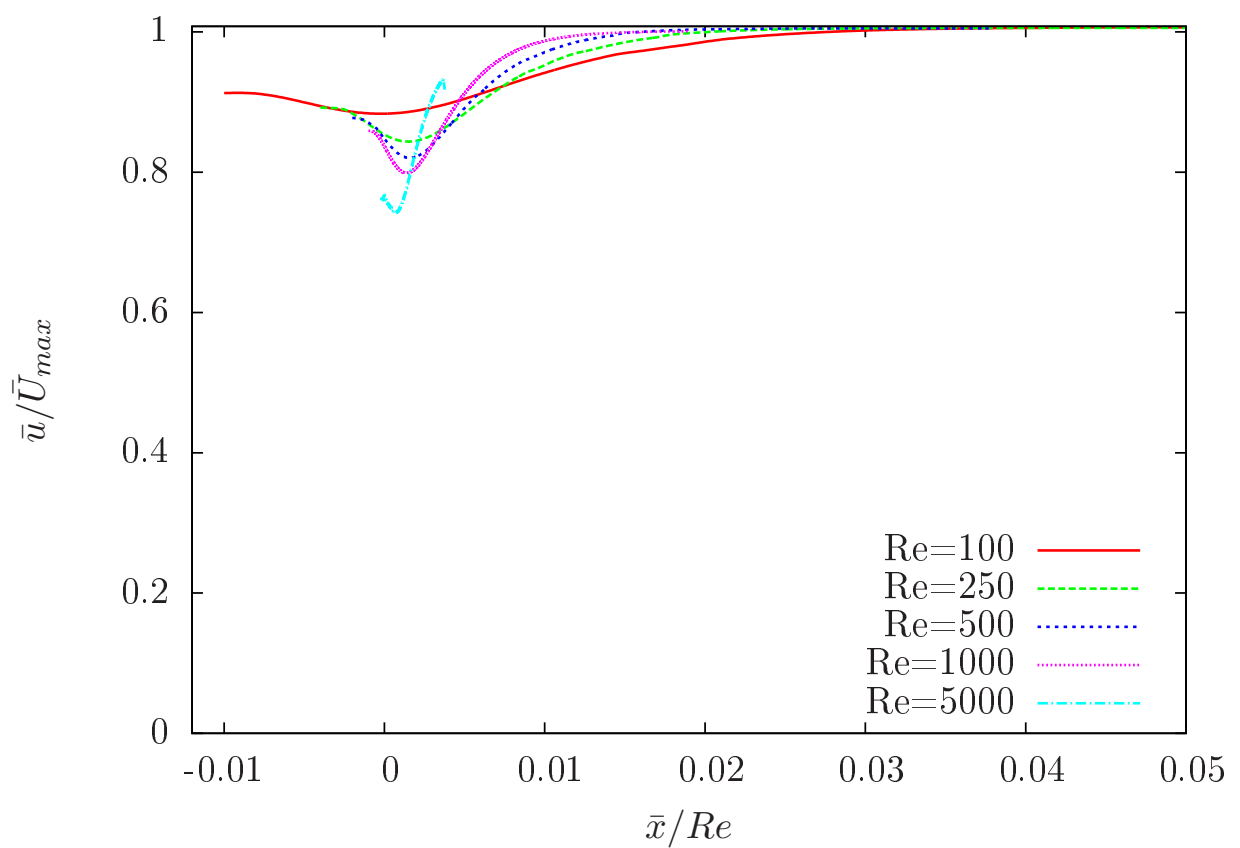

Figure 3: Numerical prediction of center line velocity $\left(\bar{u} / \bar{U}_{\max }\right.$ distribution along the axis $\bar{x} / R e$ of the pipe for several values of the Reynolds number, $\bar{\ell}=1, \bar{L}_{c}=19$. The velocity does not change so much around the value 1 . (color online) 


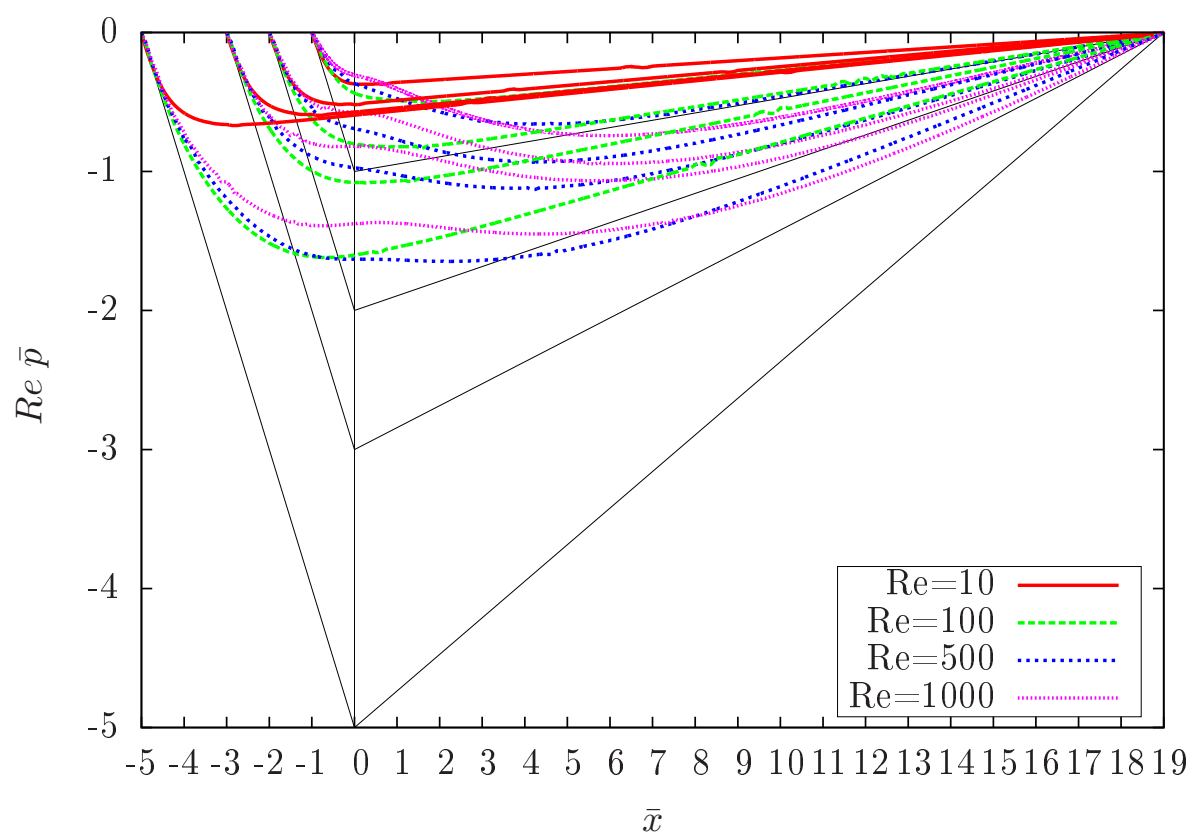

Figure 4: Numerical prediction of the distribution of pressure, scaled with the 1D prediction of the following section: $4 \bar{p}(\bar{x}, \bar{r}=0) R e / \bar{U}_{\max }$ where $T_{m}$ is the mean value of temperature 7 along the axis of the pipe of length $\bar{L}_{c}=19$ for several Reynolds numbers $(R e=10,100,500,1000)$ and for various collector length $\bar{\ell}=1,2,3,5$. The black lines correspond to the Poiseuille pressure corresponding to the value of the flux (color online). 


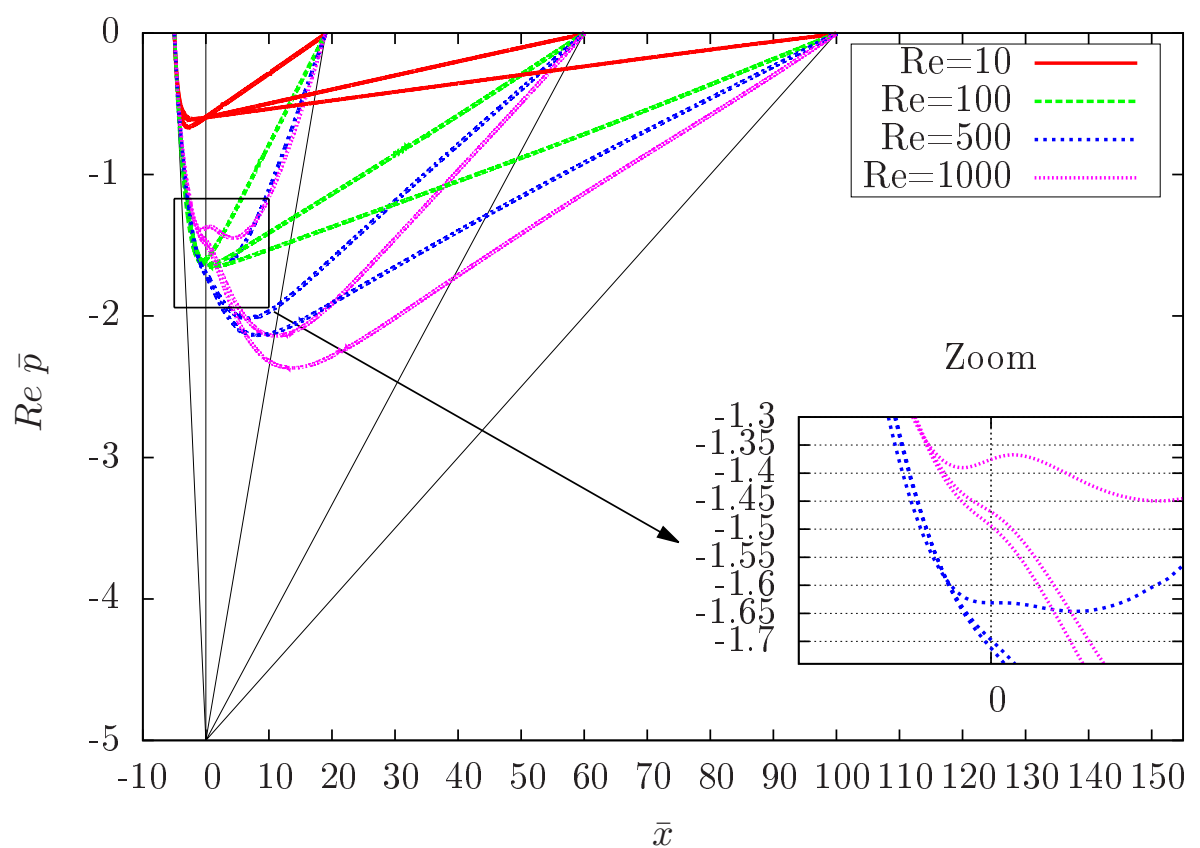

Figure 5: Numerical prediction of the distribution of pressure times Reynolds $\bar{p}(\bar{x}, \bar{r}=0) R e$ along the axis of the pipe with a collector of length $\bar{\ell}=5$ for several Reynolds numbers $(R e=10,100,500,1000)$ and for various length of the pipe $\bar{L}_{c}=19,60,100$ (color online). 


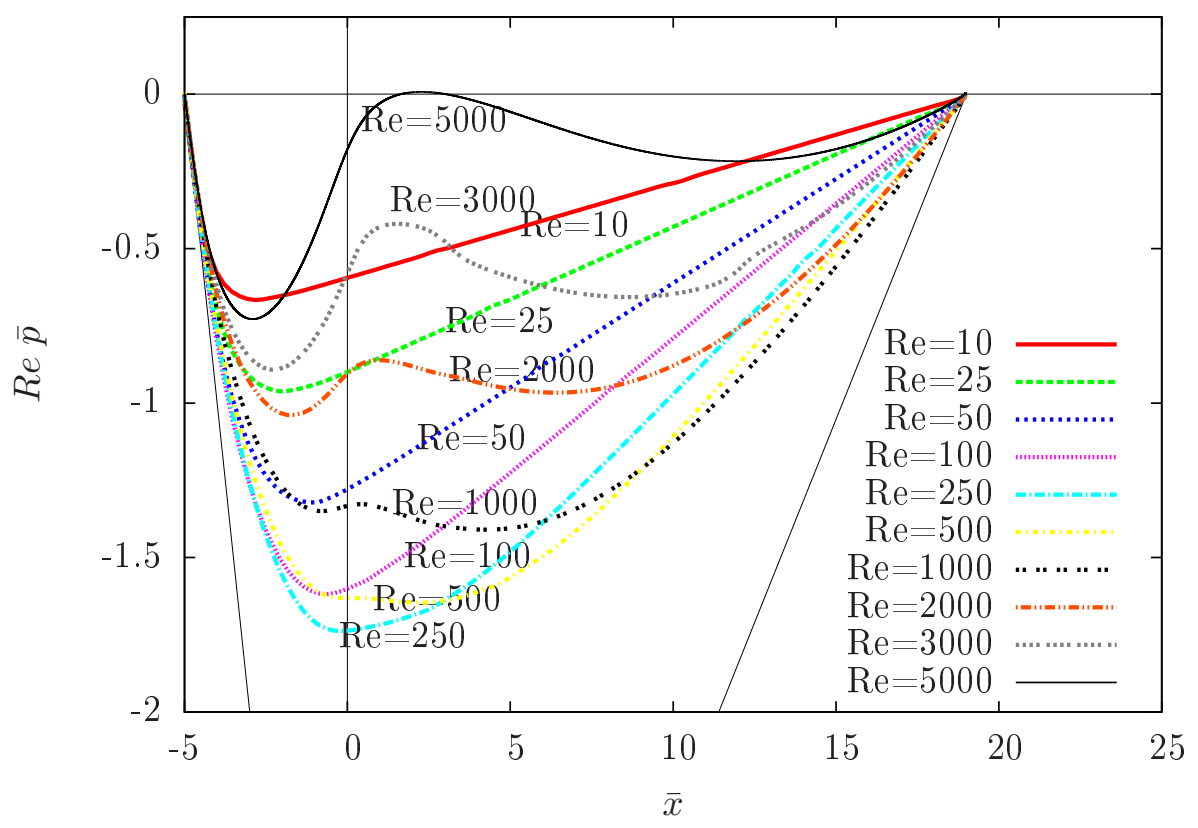

Figure 6: Numerical prediction of the distribution of pressure $\operatorname{Re} \bar{p}(\bar{x}, \bar{r}=0)$ along the axis of the pipe with a collector of length $\bar{\ell}=5$ for a length of the pipe $\bar{L}_{c}=19$. When increasing the Reynolds number, note the smooth curves for $R e=10,25,50$ and 100. and the development of a hump for $250<R e$. For $R e>5000$, a positive maximum of pressure appears and the flow becomes unsteady (color online). 

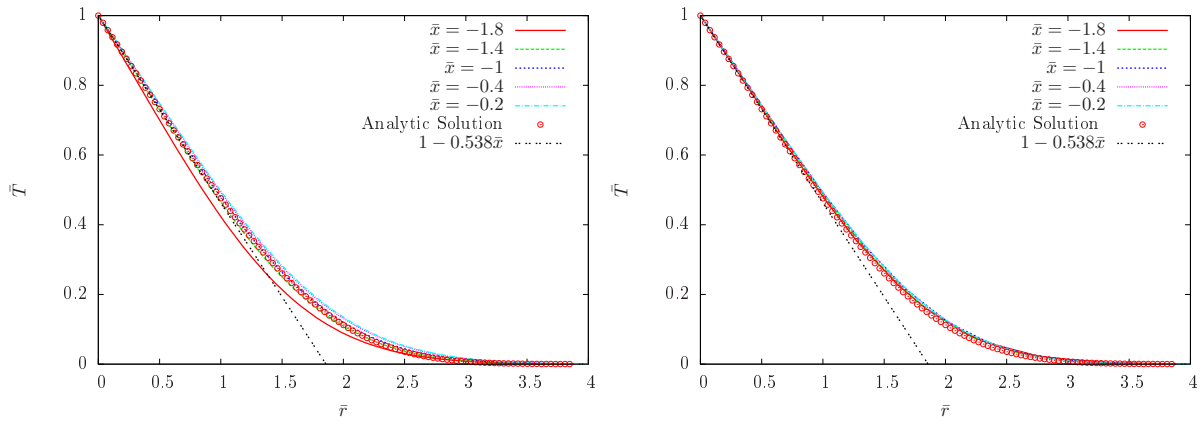

Figure 7: Numerical transverse profiles of temperature at $R e=510^{3}, L_{c}=19$, $\bar{\ell}=2$, at position $-1.8-1.4-1.0-0.4-0.2$, left for a Poiseuille flow, right for the buoyant flow in the chimney the temperature is plotted with the self similar variable $\eta$ of the Lévêque analytical solution $\theta(\eta)=\Gamma\left(1 / 3, \eta^{3} / 9\right) / \Gamma(1 / 3)$, The numerical solution of the full problem behaves as the solution of the asymptotic self similar Lévêque problem (color online). 


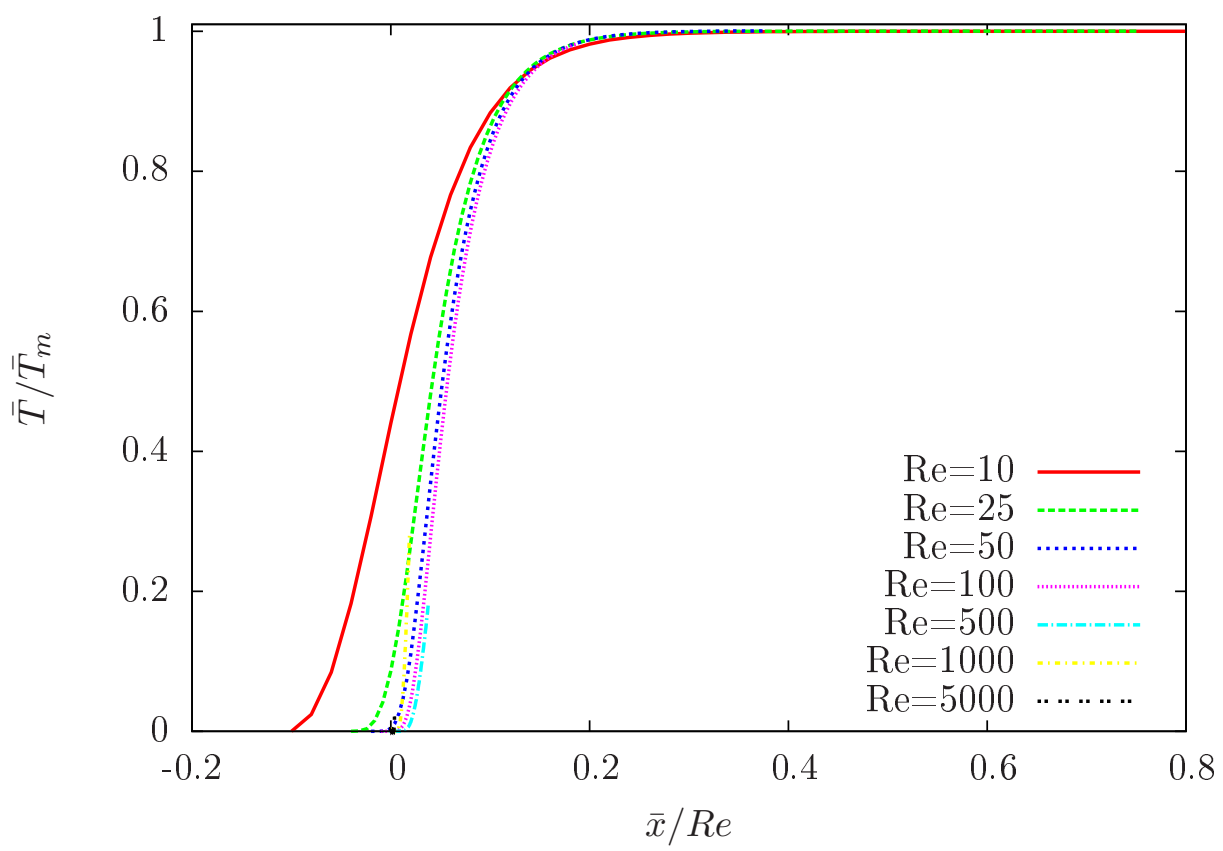

Figure 8: Temperature at the center of the pipe as function of $\tilde{x}=\bar{x} / R e$ for various $R e$. The numerical solution of the full problem behaves as the solution of the asymptotic Graetz problem as all the curve collapse on the same curve function of $\tilde{x}=\bar{x} / R e$, for $R e>25$, case $\bar{\ell}=1, \bar{L}_{c}=19$ (color online). 


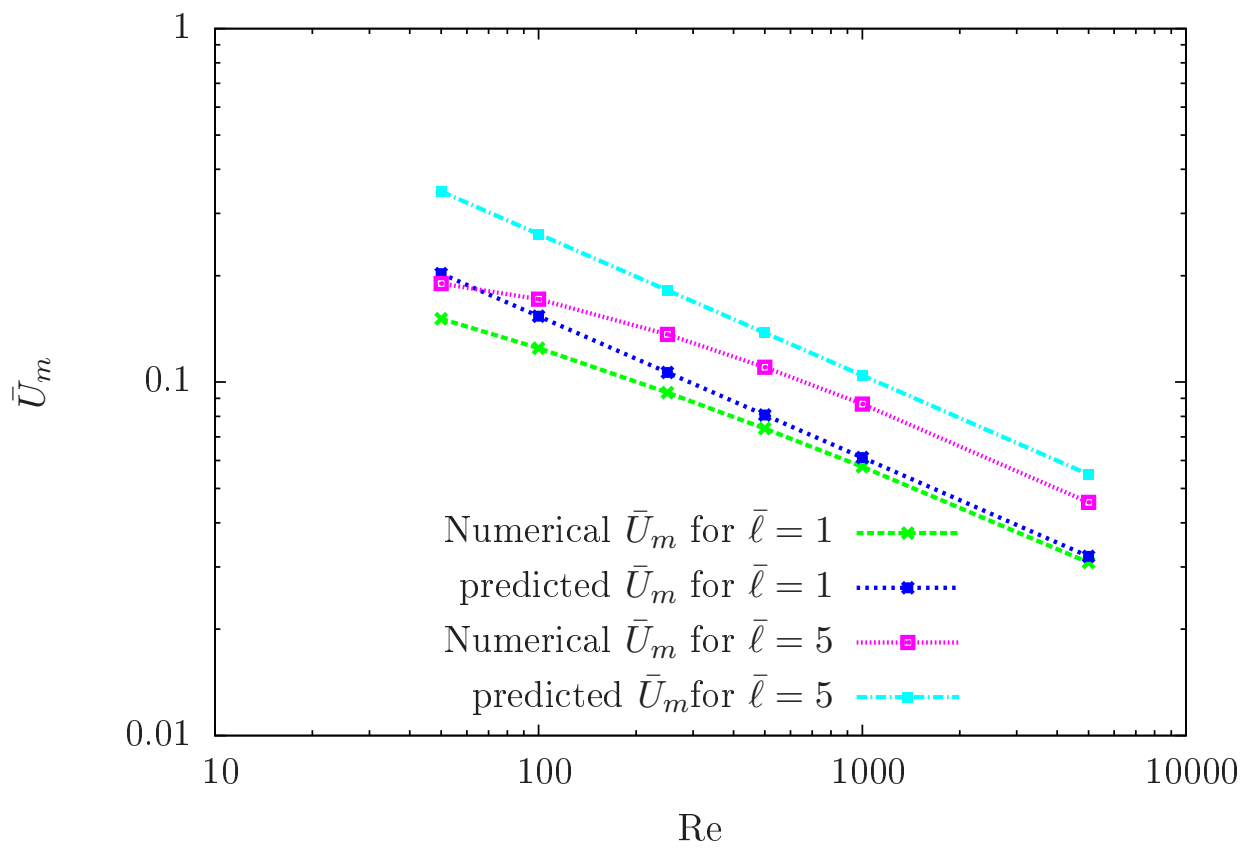

Figure 9: Prediction of the value of $\bar{U}_{\max }$ as a function of $R e \bar{\ell}$ and $\bar{L}_{c}$ compared to numerical simulations $\bar{\ell}=1, \bar{L}_{c}=19$ and $\bar{\ell}=5, \bar{L}_{c}=19$ (color online) 


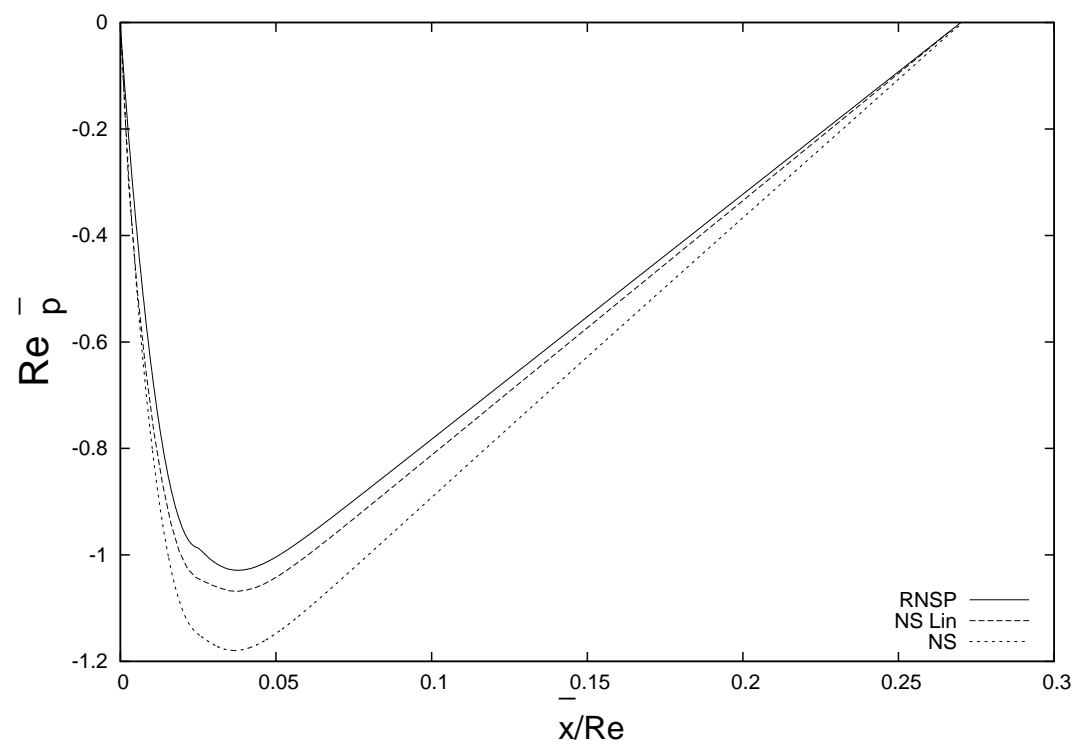

Figure 10: Pressure $R e \bar{p}$ as function of $\bar{x} / R e$, model; $\bar{\ell}=5 \bar{L}_{c}=49 R e=200$. From lower to upper curve: computed value NS $\bar{U}_{\max }=0.167$, Finite elements Linearised NS $\bar{U}_{\max }=0.172$, for RNSP $\bar{U}_{\max }=0.1747$. The decrease and the increase are smooth. 


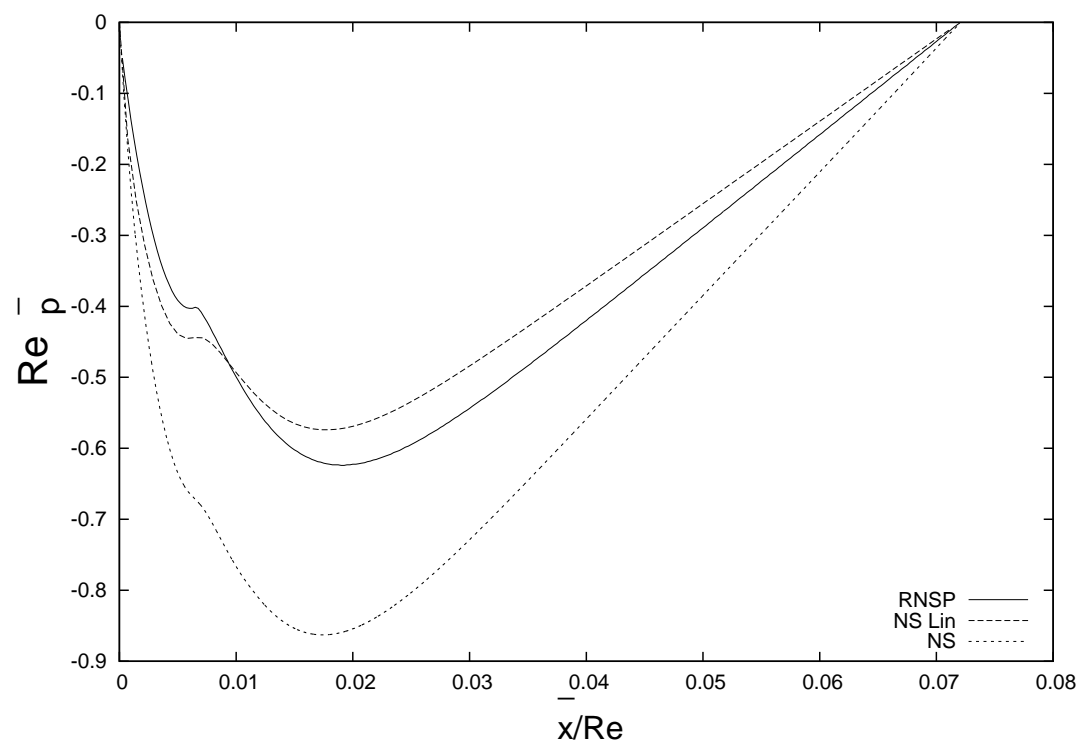

Figure 11: Pressure $R e \bar{p}$ as function of $\bar{x} / R e$, model; $\bar{\ell}=5 \bar{L}_{c}=49 R e=$ 750. A hump appears in the decreasing part and then the increase is smooth. From lower to upper curve: computed value NS $\bar{U}_{\max }=0.109$, Finite elements Linearised NS $\bar{U}_{\max }=0.10$, for RNSP $\bar{U}_{\max }=0.11$. The overall pressure shape is reproduced. 


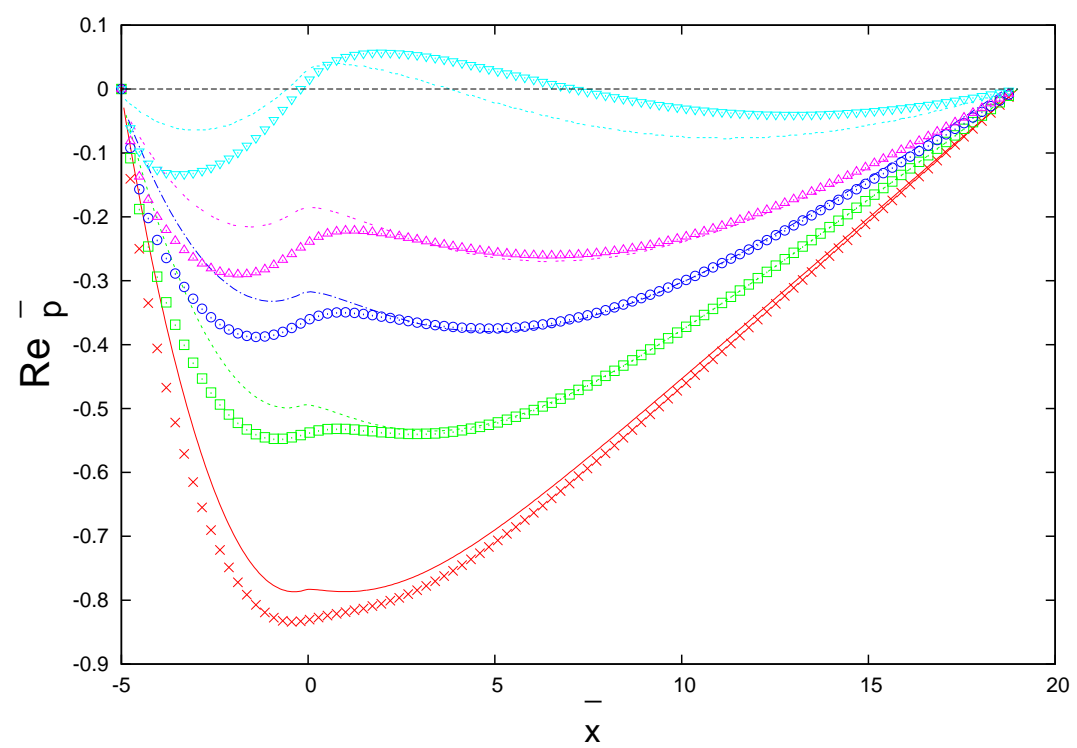

Figure 12: Pressure $\bar{p} R e$ as function of $\bar{x} ; \bar{\ell}=5 \bar{L}_{c}=19$, from bottom to top $R e=250,500,750,1000$ and 2000, dotted lines: Linearised Navier Stokes, symbols RNSP. Note the good qualitative behavior compared to the Navier Stokes (color online). 